\title{
Production of Bioactive Nanomaterial Using Laser Generated Nanoparticles
}

\author{
Anne HAHN ${ }^{1}$ and Stephan BARCIKOWSKI ${ }^{1}$ \\ ${ }^{1}$ Laser Zentrum Hannover e.V., Hollerithallee 8,30419 Hannover, Germany \\ E-mail: A.Hahn@lzh.de
}

\begin{abstract}
Laser ablation is demonstrated as an effective tool to generate highly pure nanoparticles in liquids to design novel bioactive materials. In our study we present a novel route to design bioactive materials addressing two bioactive effects via nanocomposites and nanosurfaces. Nanoparticles are generated during laser ablation and are characterised using environmental scanning electron microscopy, element mapping (energy dispersive X-ray spectroscopy) and transmission electron microscopy. The nanoparticle size, productivity and in-situ functionalisation is investigated in ethylacetate as carrier fluid for embedding nanoparticles into polymers. In-situ functionalisation in $0.01 \%$ and $0.1 \%$ solution of silicone resin leads to an increase of silver-nanoparticle productivity. Furthermore, we found that NiTi-nanoparticles are generated stoichiometrically from the bulk and that cells adhere onto nanosurfaces coated with a nanoparticle concentration of $1 \mu \mathrm{g} / \mathrm{cm}^{2}$. Overall, we present a laser-based access to two types of bioactive nanomaterials.
\end{abstract}

DOI: 10.2961/jlmn.2009.01.0010

Keywords: Bioactive nanomaterials, nanoparticles, laser ablation, drug release

\section{Introduction}

In recent years the use of nanotechnology has shown to be one of the most promising fields to enhance material properties for instance for biomedical applications [1,2]. Using nanoparticles is one route to design novel bioactive materials [3]. Synthesis of nanoparticles is often based on chemical processes. These methods show limitations for instance in the availability of materials, the dispersion of the nanoparticles, agglomeration and impurities. In particular impurities are intolerable for their use in biological systems.

Laser ablation is demonstrated as an effective alternative tool to generate highly pure nanoparticles in a variety of liquids [4]. This technique enables a nanoparticle generation of nearly unlimited variety of metals. Since the choice of liquid in which the nanoparticles are generated is variable, the handling of further process steps is facilitated.

In this study, nanoparticles generated using laser ablation in liquids is used to design bioactive materials. Depending on the intended biological effect, two nanofunctionalised materials are examined: nanomodified surfaces and bioactive nanocomposites. Surface modifications address nanomechanical effects while nanocomposites use biochemical effects of released metal ions (drug delivery).

\subsection{Surface modification}

The addressed effect of a nanomodified surface based on nanomechanics. Studies revealed that nanostructured surfaces in comparison to microtexturised surfaces enhance cell proliferation [5]. The aim therefore is to design implants with a surface modification to enable an optimized ingrowth. Cell attachment as well as cell adherence is the main focus to improve such implant materials. The approach of this study is to coat implant surfaces with nanoparticles consisting of the same material than the implant itself in order to avoid gradients of elasticity or tensile strength between core material and coating. Shape memory alloys as implant material in reconstructive surgery are gaining increasing interest. Until now, laser ablation in liquid is known as only method to generate colloidal shape memory alloy nanoparticles [4]. In this study we examine the generation of Nickel-Titanium-shape memory alloy nanoparticles for surface coatings.

\subsection{Nanocomposites}

The second group of nano-functionalised materials address biochemical effects which is realized by the generation of bioactive polymeric composites with embedded metallic nanoparticles. The bioactivity is based on a drug release effect where metallic nanoparticles degrade and metal ions diffuse through the polymer into the surrounding media or biological system, respectively $[3,6]$. In order to generate bioactive nanomaterials as drug release system e.g. such as cochlea implants with combined effects on different cell types like antiproliferative and neurotroph effects various elements may be embedded into an implant material.

The concentration of the metal ion release depends on a variety of parameters e.g. the size of embedded nanoparticles and their filling rate. In order to avoid local peak concentrations a homogenously distribution of nanoparticles in the polymer matrix should be achieved. Thus, agglomeration during the process steps should be prevented. Furthermore, the coupling of the nanoparticles plays an important role and needs to be examined from the first process step on. In our study we examine the nanoparticles from their generation to the embedding in the solid polymer.

\section{Experimentals}

Nanoparticles used for both bioactive effects using nanocomposites and nanosurfaces are generated during laser ablation in liquids and are characterised using envi- 
ronmental scanning electron microscopy (E-SEM), transmission electron microscopy (TEM), element mapping using energy dispersive X-ray spectroscopy (EDX) and energy electron low spectroscopy (EELS). NiTi and silver are used as targets and are placed in a reservoir of ethylacetate, n-hexane and acetone, respectively. The laser beam (pulse duration: 120 fs, wavelength: $800 \mathrm{~nm}$, average power: $2.5 \mathrm{~W}$; repetition rate: $5 \mathrm{kHz}$ ) is guided on the surface of the material. Self focussing in the liquid leads towards a filamentation and an ablation of the material.

NiTi nanoparticle colloid dispersed in acetone was dropped on a glass substrate resulting in a concentration of $1 \mu \mathrm{g} / \mathrm{cm}^{2}$. Silver nanoparticles are generated in ethylacetate doped with silicone resin or curing agent. Silicone resin and curing agent were commercially available as two component system. After vaporisation of the organic solvent the nanoparticle suspension is polymerised.

\section{Results}

\subsection{Surface modification}

Nanoparticles generated during laser ablation of NiTi foil in acetone are shown in Fig. 1 using TEM analysis. The nanoparticles have been embedded into a solid matrix. Nanoparticles are spherical and stable within this matrix. The Ferret diameter is in the range of 30 to $80 \mathrm{~nm}$. An element mapping of the marked red spot on the left image was carried out to analyse the composition of the nanoparticles ablated from NiTi-alloy. Element mapping reveals both elements Nickel and Titanium in this spot as well as in the single $60 \mathrm{~nm}$ particle. Consequently, NiTi-alloynanoparticles have been generated.

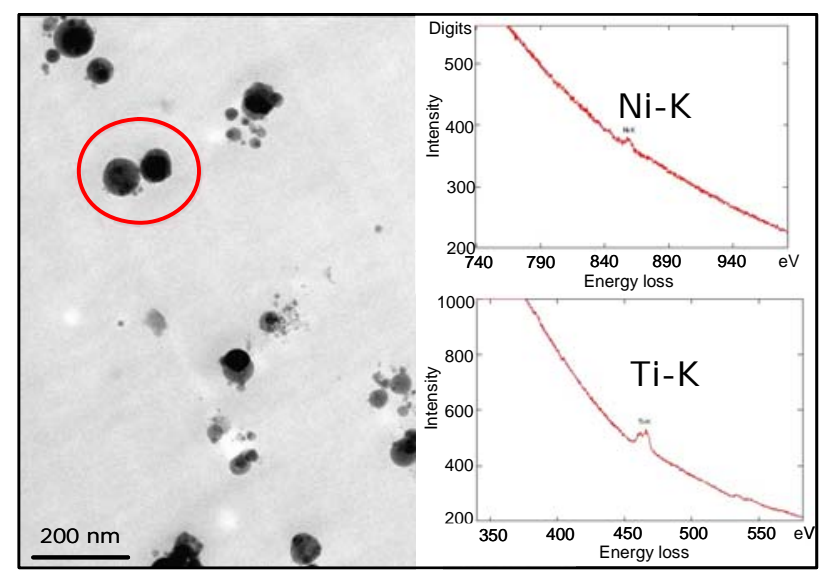

Fig. 1. Transmission electron microscopy of NiTi nanoparticles generated during laser ablation in acetone (left) and electron energy loss spectroscopy of marked red spot (right).

Despite the positive TEM analysis we cannot fully exclude that during the nanoparticle generation process Nickel and Titanium nanoparticles are additionally generated. Nickel is known to cause allergic reactions [7]. Therefore this might lead to adverse effects on cells. On the surface of the materials the highest concentration of metal ions is found. Metal ions have an influence on cells when the concentration reaches toxic levels. At the materials surface the concentrations are higher than in the surrounding cell culture media. Therefore an effect on the attachment of the cells on the material itself might occur. Consequently, NiTi-nanoparticles have been brought on a glass surface via vaporization of the solvent from the surface with a final concentration of $1 \mu \mathrm{g} / \mathrm{cm}^{2}$. Osteoblasts, cells which are responsible for bone formation, have been cultivated on this substrate. In Fig. 2 an E-SEM image of this cultivated surface is shown. The dark areas in the images display the cells whereas the white spots are NiTi-nanoparticles. The grey area is the glass background.

The picture shows that nearby the cells a porous matrix has been developed. White spots are placed within this matrix. It can be seen that the dark areas are spread over the matrix.

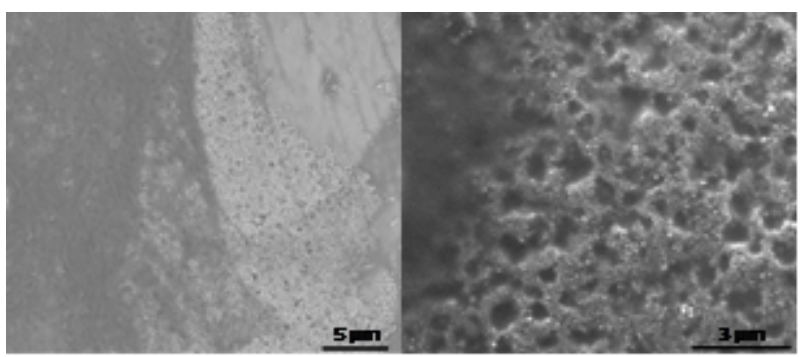

Fig. 2. Environmental scanning electron microscopy of osteoblasts cultivated on a nanoparticle coated glass substrate.

It can be assumed that the cells have developed an extra cellular matrix or that the nanoparticles are coated with organic residues. The images show an intact cell morphology. All in all we found that a NiTi-nanoparticles concentration of $1 \mu \mathrm{g} / \mathrm{cm}^{2}$ do not have any adverse effect on osteblasts. Cell extensions are grown over the nanoparticles coating and most important cell attachment could be established.

We demonstrated first steps of designing bioactive nanomaterials using surface functionalisation. In the following a modification of nanomaterials in three dimensions is studied based on a functionalisation within the volume of the material.

\subsection{Nanocomposite fabrication for drug release sys- tems}

Drug release systems are based on the release of agents out of a material. Metal ion release caused by slow oxidation (biocorrosion) of nanoparticles within a polymeric matrix is used to achieve a continuous release.

Silver and copper nanoparticles are well known to cause antiproliferative effects [8]. In this investigation the embedding of silver nanoparticles into silicone is demonstrated.

It has to be ensured that the nanoparticle distribution within the polymer is homogenous. Peak concentrations based on agglomerates at different locations in the matrix could have unwanted side-effects. In order to ensure a homogenous distribution of nanoparticles in the polymer a coupling of the nanoparticles in the matrix has to be achieved. This can be realized using an in-situ functionali- 
sation during the laser ablation process. We studied therefore the influence of silicone resin and curing agent as additives during the nanoparticle generation process. Since the stabilization has an influence on the nanoparticle ablation rate [9], different rates of silicone were added to ethylacetate. The mass ablation rate was measured and shown in Fig. 3. An increase of the nanoparticle production rate while adding 0.01 to $0.1 \%$ of silicone to the solvent has been revealed. The highest nanoparticle production rate of $1.3 \mathrm{mg} / \mathrm{h}$ is observed using $0.01 \%$ of silicone which results in a $65 \mu \mathrm{g} / \mathrm{ml}$ concentration of silver nanoparticles in ethylacetate. The images of the samples are shown in the insert. Using $0.01 \%$ of silicone resin leads towards a darker yellow dispersion than using different rates of silicone resin. The UV-VIS spectra of the samples were measured and shown in Fig. 4. The highest absorption is contributed to silver nanoparticles generated in ethylacetate with $0.01 \%$ of silicone which corresponds to the high nanoparticle productivity in this range. The absorption spectrum of the particles generated in $0.01 \%$ to $0.1 \%$ exhibit a second peak in the range of $530 \mathrm{~nm}$ which is characteristic for occurring agglomerates. There is evidence that the concentration of nanoparticles within the solution has achieved a saturation level and agglomeration takes place.

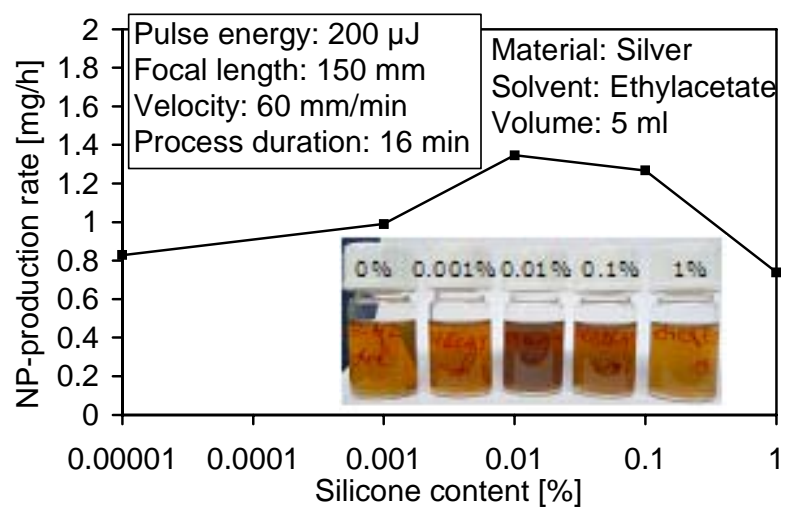

Fig. 3. Influence of silicone content on the mass ablation of silver in ethylacetate

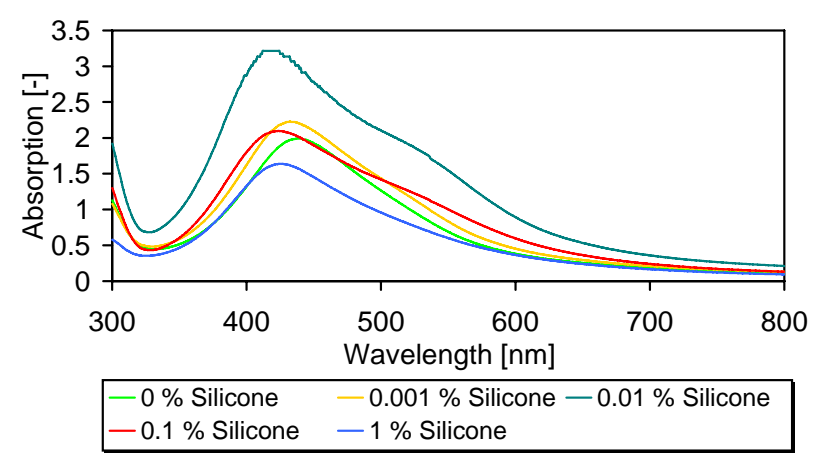

Fig. 4. Absorption spectrum of silver nanoparticles generated in different contents of silicone added to ethylacetate.

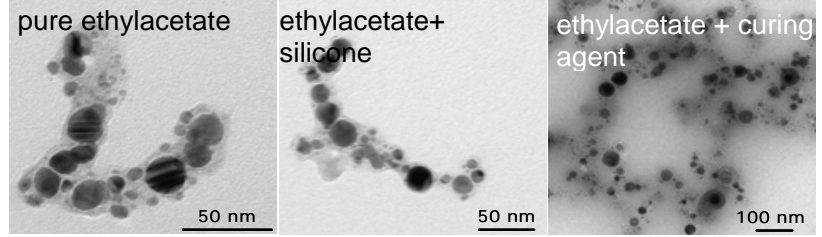

Fig. 5. Transmission electron microscopy of silver nanoparticles generated in pure ethylacetate, ethylacetate doped with silicone and with curing agent.

Fig 5. shows TEM images of silver nanoparticles generated in pure ethylacetate, doped with silicone resin and curing agent, respectively. A shell around the nanoparticles formation has been developed in case of pure ethylacetate and ethylacetate doped with silicone. This shell might disturb the coupling within the polymer matrix. In order to ensure a coupling of the nanoparticles within the polymeric matrix, ethylacetate doped with curing agent seems to have the highest potential. The nanoparticles are linked to each other. No formation of residual shell around the nanoparticles is seen. Therefore, we assume that a coupling with polymeric matrix is realized. All in all, ethylacetate as solvent has a high affinity towards the generated nanoparticles and has to be substituted by active linkers. The next steps will be to vary the solvent to enhance the linking of nanoparticles within the polymer.

\section{Conclusion}

The generation of nanoparticles during laser ablation in liquids has been demonstrated as an effective tool giving access to nanomaterials for medical application. NiTinanoparticles have been generated during ablation of NiTialloy. Both elements have been detected in single nanoparticle, so that a stoichiometrically conversion is assumed. Cell attachment has been observed on a surface coated with laser generated NiTi-nanoparticle with a concentration of $1 \mu \mathrm{g} / \mathrm{cm}^{2}$. The next step will be to increase the surface concentration and to study the effect of nanoparticles on a NiTi implant material.

The design of nanomaterials as metal ion release systems using a volume functionalisation within the material has been studied. In-situ-functionalisation has been examined using silicone contents of $0.01 \%$ and $0.1 \%$ added to the generation process in ethylacetate and an increase of the nanoparticle production rate to a value of $1.4 \mathrm{mg} / \mathrm{h}$ has been shown. However, our investigations found out that ethylacetate has a high affinity to silver nanoparticles. The in-situ functionalisation using dissolved curing agent in the liquid matrix before the ablation process seems to bear the highest potential to enhance the homogenous distribution within the matrix.

In summary, first steps towards the generation of bioactive nanomaterials using laser generated nanoparticles have been demonstrated for material surface and volume functionalisation. 


\section{Acknowledgment}

The authors acknowledge the German Research Foundation for funding this work within the Collaborative Research Centre TransRegio 37 "Micro- and Nanosystems in Medizine - Reconstruction of biologic Functions". Additionally, authors thank Swen Maas of the Material Analytischer Service in Freiburg for carrying out the transmission electron microscope images.

\section{References}

[1] L.E. Bromberg, V.M. Braman, D.M. Rothstein, P. Spacciapoli, S.M. O’Connor, E.J. Nelson, D.K. Buxton, M.S. Tonetti, P.M. Friden. Journal of controlled release, 68 (2000), $63-72$.

[2] T. Maneerung, S. Tokura, R. Rujiravanit. Carbohydrate Polymers, 72 (2008), $43-51$.

[3] C. Damm, H. Münstedt, A. Rösch. Materials Chemistry and Physics, 108 (2008), 61 - 66.

[4] S. Barcikowski, M. Hustedt, B. Chichkov. Polimery, 53 (2008), $657-662$.

[5] S. Choudhary, K.M. Haberstroh, T.J. Webster. Tissue Engineering, 13 (2007), 1421 - 1430.

[6] S. Cai, X. Xia, C. Xie. Biomaterials, 26 (2005), 26712676.

[7] T. Habijan, O. Bremm, S.A. Esenwein, G. Muhr, M. Köller. Materialwissenschaft und Werkstofftechnik, 38 (2007), 969 - 974.

[8] F. Heidenau, W. Mittelmeier, R. Detsch, M. Haenle, F. Stenzel, G. Ziegler, H. Gollwitzer. J. Mater. Sci. Mater. Med., 16 (2005), 883-888.

[9] S. Barcikowski, J. Jakobi, S. Petersen, A. Hahn, N. Bärsch, B. Chichkov. In: Proceedings of 24nd International Conference on Applications of Lasers and Electro-Optics ICALEO, $10 / 29 / 2007$ to $11 / 01 / 2007$, Orlando, FL, USA. 108-113.

(Received: June 16, 2008, Accepted: March 10, 2009) 